\section{Ion Beams in Art and Archaeology}

\author{
G. Demortier from the Laboratoire d'Analyses par Réactions Nucleaire (LARN), Namur, who \\ chairs the COST Action G1 (Ion-Beam Analysis Applied to Art and Archaeology) summarises the \\ advantages of using accelerator-based techniques to study artifacts.
}

The application of analytical techniques - initially developed in the field of materials science - to art or archeological objects gives the historian and the archaeologist quantitative information. This helps them understand the way of life in the cultures they are studying, or the technical and intellectual know-how of the artists or craftsmen of the period under consideration. In the world of musea, this knowledge is also needed for preparing and carrying out essential restorations, and for assessing the authenticity of artefacts and paintings proposed for acquisition. Furthermore, our entire cultural heritage is more or less doomed to disappear owing to ageing and the deleterious effects of pollution. The degradation of monuments and outdoor statues is well known, but all artefacts, even those in exhibits or storage rooms, are subject to degradation. The phenomena must be studied to understand their cause and their kinetics, and to find out ways to slow them down or to prevent them taking place.

All these objectives are common to the very large community of those who practice what is called today "archaeometry" or more generally "application of sciences to art". Much of this work is based on elemental analysis, namely determining the concentration of elements present in an object. Until the 1960 s, only chemical techniques were available for analyzing composition. Since then, many others methods based on physical principles have beein developed. They include microanalysis using electron beam microscopes and microprobes and approaches based on various types of particle and photon spectrometers. This interest led to the construction of major accelerator-based facilities devoted to archaeometry such as the AGLAE accelerator at the Louvre (see photograph). The insert describes the principle accelerator-based techniques used for elemental analysis and their relative advantages.

More recently, methods based on the use of accelerated ions - so-called ionbeam analysis - have developed rapidly. To further exploit this approach it was decided in the early-1990s to launch a

The Action coordinates its scientific cooperative COST action called Ion Beam Analysis Applied to Art and Archaeology. A memorandum of understanding for a COST Action was signed in June 1994, and the concerted scientific activities started in January 1995. The goals were as follows: - The development of non-destructive nuclear and atomic methods for studying items of art and archaeology using particle irradiation.

- The comparison of these novel techniques with conventional methods already used in museum laboratories for examining objects of art.

- The creation of an interdisciplinary forum of scientists involved in archaeology and archaemetry. activities among the participating groups (see table) by organizing the exchange of samples and procedures. A second aspect is to arrange for specialists to work together for a short time, in a particular laboratory, on artefacts of archeological interest provided by the participating laboratories. Priority is given to the study of artefacts proposed by archaeologists and historians who define the archeological pertinence of the analytical work.

The Action also organizes workshops and experimental sessions on the concerted research at the rate of two meetings each year. Since the Action started there
COST GI Participating Laboratories

Underlined are the groups which are working with ionbeam facilities. Collaborations also exist with experts based outside Europe (Delaware, USA; Lower Hutt, New Zealand; Faure, South Africa; Lucas Heights, Australia).

AUSTRIA

Vienna: Akademie der Bildenden Kunste

BELGIUM

Namur: LARN, Facultés Universitaires ND de la Paix

Antwerp: MITAC, Universitaire Instellingen

Liège: Université

FINLAND

Helsinki: Helsingin Teknillinen Oppilaitos

Helsinki: Finnish National Gallery

FRANCE

Paris: Laboratoire de Recherche des Musées de France

Orléans: Centre Ernest Babelon, CNRS

GERMANY

Dresden: Forschungszentrum Rossendörf

Berlin: Hahn-Meitner-Institut

GREECE

Athens: NCSR Demokritos

HUNGARY

Debrecen: ATOMKI

Budapest: KFKI Institute for Particle and Nuclear Physics ITALY

Arcetri-Firenze: Università degli Studi

Venice: Università degli Studi

ROMANIA

Bucharest: Institute of Atomic Physics

SLOVENIA

Ljubljana: Institut Josef Stefan

SPAIN

Madrid, Universidad Autonoma

Sevilla, Universidad

UK

Oxford: SPM, University of Oxford

have been workshops and seminars in Debrecen (on general aspects of using ionbeam techniques to study archeological artefacts), in Sevilla (with talks on ionbeam techniques for historians and archaeologists), in Dresden (on the simulation of the depth-profiling analysis of pigments), and in Paris (on problems of conservation in musea). The next work-

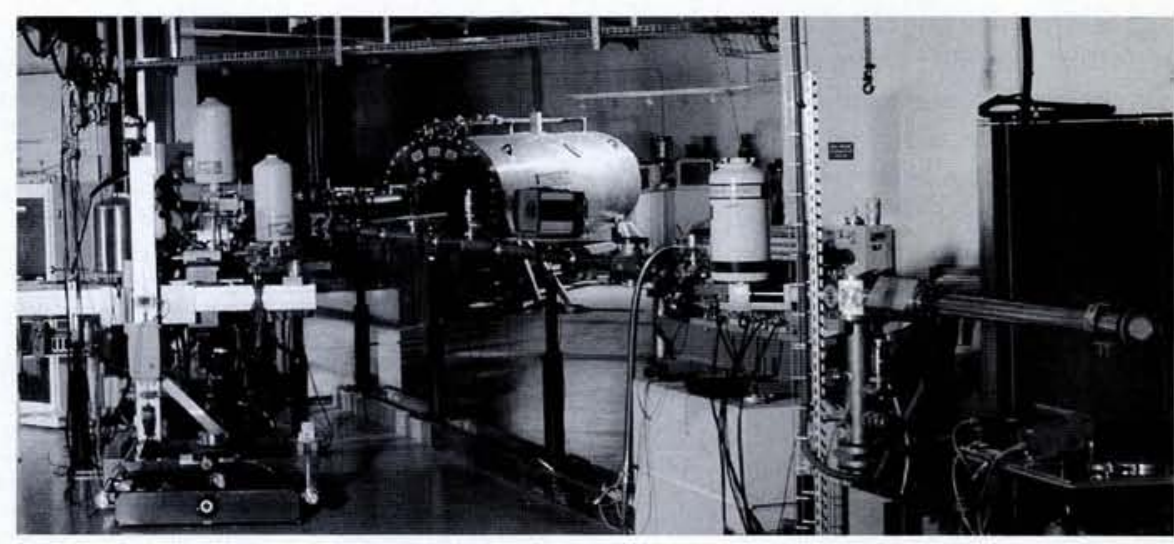

The Accelerator Grand Louvre pour l'Analyse Elémentaire (AGLAE) at the Louvre Museum, Paris. The right-hand side of the photograph shows the external beam assembly that allows the study of large artifacts under ambient conditions. The AGLAE laboratory was set up at the end of the 1980 s mainly to provide within the museum itself powerful analytical tools for a large community of historians and archaeologists. COST G1 groups from Florence, Namur, Orléans, and Oxford have collaborated with the laboratory for many years. 


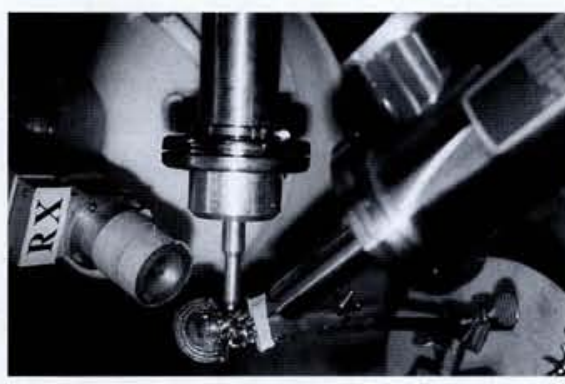

A photograph of the experimental arrangement at LARN's ambient conditions PIXE facility in Namur, Belgium, showing the relative positions of instruments. The X-ray detector for collecting signals from argon (in air) is seen in the upper part while the lower part shows the main X-ray detector for identifying the elements present in the artefact under examination. The artefact (seen in the middle of the photograph) is an anthropomorphic pendant from the pre-Hispanic period which is positioned for analysis at the exit window of the evacuated accelerator beam pipe.

shop, to be held in Namur in spring 1997, will focus on the damage that can arise in fragile materials on exposure to ion-beam irradiation.

Among the scientific collaborations already established, one should include: - the depth-profile analysis of the layered structures of paintings (initiated by the Dresden group and involving a major collaboration with the groups in Vienna, Paris, Namur, Liege, Sevilla, and Florence); - the study of the technology of ancient glasses (proposed by the Debrecen and Orléans groups);

- the preparation of laboratory standards for the study of papers, pigments and inks (Oxford and Dresden groups);

- the elemental analysis of glass and enamel on glass using particle-induced X-ray emission and particle-induced gamma-ray emission (Dresden and Vienna groups); - an attempt at a non-destructive characterization of bronzes from various areas of the Iberian peninsula and Belgium (Madrid, Liège and Namur groups); - two studies of gold jewellery artifacts (Namur, Orléans and Sevilla groups); - a long-term collaboration on the general use of ion beams for non-vacuum and vacuum microprobe elemental characterization of a large variety of museum objects (Oxford, Paris and Florence groups).

\section{Further Information}

For further details and information about the possibility of joining the Action, please contact one of the Coordinators of the working group or the Chairman of COST Action G1: Prof. G. Demortier, Laboratoire d'Analyses par Reactions Nucléaires, Facultes Universitaires Notre-Dame de la Paix, 22 rue Muzet, B-5000 Namu (tel.: +32-81-73 12 67; fax:

+32-81-73 79 38; e-mail: guy.demortier@fundp.ac.be)

\section{Accelerator-based Elemental Analysis}

In comparison with methods involving electron-beam irradiation, the use of heavier particles allows measurements (see table) of the composition of objects under ambient conditions, a feature which is of great advantage in studying fragile materials (e.g., ancient manuscripts) and large items (e.g., statues, paintings) and for rapidly selecting regions to be analyzed (e.g., inclusions in potteries; solders on gold artefacts).

The photograph on the left illustrates a typical experimental arrangement for analysis under ambient conditions employing a particle accelerator. A $0.7 \mathrm{~mm}$ in diameter proton beam is being used to analyze the various components (bulk wires, granulations, solders, etc.) on an anthropomorphic pendant made of tumbaga, an alloy with a low concentration of gold dating from the the pre-Columbian period.

\section{The Principle Accelerator-based Analysis Techniques \\ Technique}

Surface techniques $(1-400 \mu \mathrm{m})$

PIGE Particle-Induced Gamma-ray Emission 0.5 to $7 \mathrm{MeV}$

PBS Rutherford Backscattering Spectrometry 0.5 to $7 \mathrm{MeV}$

$\begin{array}{lll}\text { NRA } & \text { Nuclear Reaction Analysis } & 0.5 \text { to } 7 \mathrm{MeV} \\ \text { CPM } & \text { Charged Particle Activation Analysis } & 10 \text { to } 20 \mathrm{MeV}\end{array}$

Bulk analysis

FNAA Fast Neutron Activation Analysis 10 to $20 \mathrm{MeV}$

10 to $20 \mathrm{MeV} \quad$ Elemental analysis of 60 elements among medium \& heavy ones
PIXE Particle-Induced X-ray Emission 0.5 to $7 \mathrm{MeV}$

\section{COST G1: Organization and Management}

As archaeological items and works of art cover a very large variety of materials, six working groups have been organized, whose details are given below (together with a list of laboratories having expertise and publications in the various fields.

\section{PAINT LAYERS}

Coordinator: Ch. Neelmeijer, Dresden

Tel +49-351-5913254

Fax +49-351-5913285

Paintings: Dresden, Florence, Helsinki, Le Louvre (Paris) , Ljubljana, Namur, Orléans, Oxford, Sevilla, Vienna

Manuscripts: Dresden, Florence, Le Louvre (Paris), Helsinki, Madrid, Orléans, Oxford

\section{ORGANIC MATERIALS}

Coordinator: G. Grime, Oxford Tel +44-186-527 3367 $\mathrm{Fax}+44-186-5273418$

Budapest, Debrecen, Le Louvre (Paris), Orléans, Oxford

\section{SILICATE MATERIALS}

Coordinator: M. Schreiner, Vienna

Tel +43-158816261

Fax +43-158816137

Pottery: Antwerp, Athens, Debrecen, Helsinki, Madrid, Namur, Sevilla, Venice

Glass: Bucharest, Debrecen, Le Louvre (Paris), Namur, Orléans, Vienna Flints: Ljubljana, Le Loovre (Paris) Stones: Antwerp, Le Louvre (Paris)

\section{CONSERVATION IN MUSEA}

Coordinator: J.-C. Dran, Paris Tel +33-1-40205405 Fax $+33-1-40733246$

Antwerp, Le Louvre (Paris)

\section{METALS}

Coordinator: J.-N. Barrandon, Orléans Tel $+33-2-38515270$ $\mathrm{Fax}+33-2-38517688$

Iron: Helsinki

Bronzes and brass: Antwerp,Budapest, Ljubljana, Madrid, Namur, Orléans, Sevilla

Silver: Helsinki, Ljubljana, Madrid, Namur, Orléans, Oxford Gold: Le Louvre (Paris), Madrid, Namur, Orlèans, Oxford, Sevilla

\section{RADIATION DAMAGE}

Coordinakor: G. Demortier, Namur Tel +32-81-731267

Fax $+32-81-737938$

Dresden, Le Louvre (Paris), Narnur

\section{EC-LARGE-SCALE FACILITY ULTRALOW TEMPERATURE FACILITY Universität Bayreuth}

Within the European Community programmes "Human Capital and Mobility" and "Training and Mobility of Resear chers" the Ultralow Temperature Facility provides support to users from EC countries and the associated countries

- to extend their research in condensed matter physics to lower temperature

- to allow them to become acquainted with low temperature technology

- to train young scientists in special fields of low temperature physics

The facility offers free access (including travel and living expenses) to its cryogenic equipment and peripherals for

- MATERIALS PREPARATION

Melting, annealing, cutting and grinding devices

- SAMPLE CHARACTERIZATION

$\mathrm{X}$-ray diffractometers, thermal analysis, and SQUID magnetometers

- TRAINING AND RESEARCH

Cryostats operating at 4 and $1.5 \mathrm{~K}$ and in fields of 6 and $8 \mathrm{~T}$

Dilution cryostats operating from 4 to $300 \mathrm{mK}$

Nuclear demagnetization cryostats above $0.5 \mathrm{mK}$

Nuclear demagnetization cryostats below $0.5 \mathrm{mK}$

The facility is devoted to research in condensed matter physics. In particular, NMR- and ultrasonic techniques are available as well as methods for specific heat, thermal and electrical resistivity experiments and magnetization measurements by AC- and SQUID-techniques. The variety of samples can range from quantum fluids and crystals to glasses, spin glasses, metals and HTSC-materials.

The facility would like to especially encourage applications by users who do not have access to such equipment in their own laboratory. For further information, please contact:

Prof. Dr. Georg Eska, Physik. Institut, Universität Bayreuth. 95440 Bayreuth

Tel.: +49-921-55-3342; FAX: +49-921-55-3647; e-mail: girgl@btp9xi.phy.uni-bayreuth.de 